\section{Structure of Dihydrodiphosphopyridine Nucleotide}

WEBER ${ }^{1}$ has recently reported on the intramolecular transfer of excitation energy in dihydrodiphosphopyridine nucleotide. In addition to the strong band having its maximum at $340 \mathrm{~m} \mu$, the excitation spectrum of the $462 \mathrm{~m} \mu$ fluorescence also shows a maximum at $260 \mathrm{~m} \mu$, a region where the absorption is due to the purine part of the molecule.

Kaplan et al. ${ }^{2}$ have identified an isomer of diphosphopyridine nucleotide which comprises 10-15 per cent of a highly purified commercial preparation of diphosphopyridine nucleotide. The isomer was characterized by optical rotation studies as the $\alpha$-glycoside of nicotinamide riboside as distinct from the biologically active $\beta$-isomer of diphosphopyridine nucleotide. 'This assignment of configuration about the $\mathrm{C}_{1}$ of ribose was substantiated by the synthetic preparation of nicotinamide mononucleotide ${ }^{3}$ to give a mixture of the $\beta$ - and $\alpha$-anomers in a $4: 1$ ratio.

The $\alpha$-isomer of diphosphopyridine nucleotide was reduced chemically with sodium dithionite ${ }^{4}$ and the product was examined spectrofluorometrically. Unlike the $\beta$-isomer, the excitation spectrum of the $462 \mathrm{~m} \mu$ fluorescence of $\alpha$-dihydrodiphosphopyridine nucleotide shows a strong band only when activated at $340 \mathrm{~m} \mu$ and not when irradiated at $260 \mathrm{~m} \mu$, indicating that excitation energy transfer is not as efficient with the $\alpha$-isomer as was observed. with the $\beta$-isomer. The $462 \mathrm{~m} \mu$ fluorescence resulting from $260 \mathrm{~m} \mu$ and $340 \mathrm{~m} \mu$ excitation of $\alpha$ - and $\beta$-dihydrodiphosphopyridine nucleotide is shown in Fig. 1.

Further evidence for the differences in interaction in the $\alpha$ - and $\beta$-isomers of dihydrodiphosphopyridine nucleotide may be found in their spectrophotometric behaviour in water and propylene glycol. Weber ${ }^{1}$ reported that the ratio $E_{260} / E_{340}$ is 2.85 for the

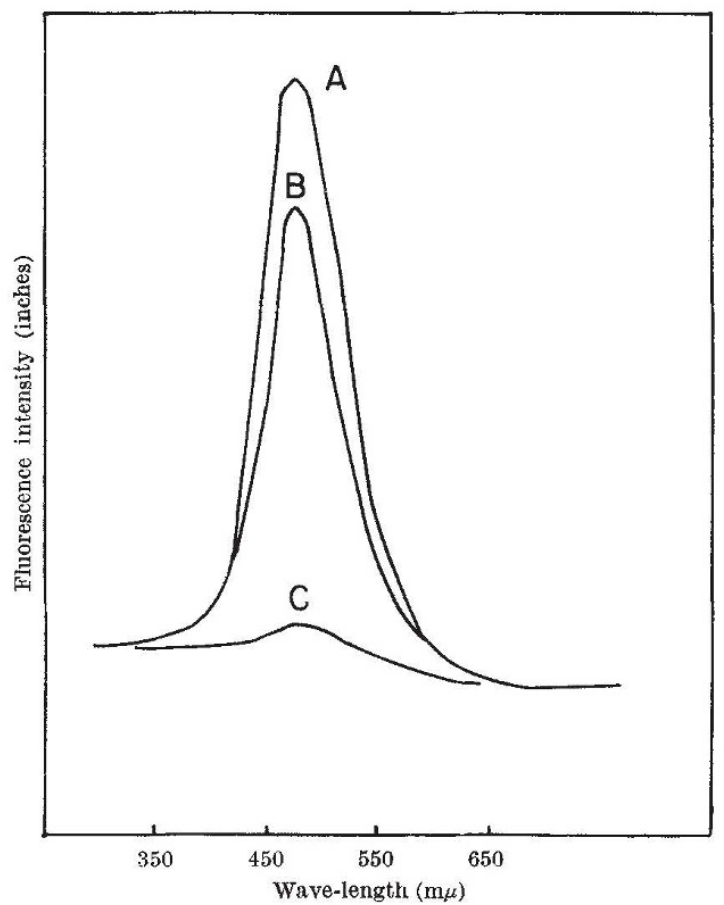

Fig. 1. Fluorescence spectra of $\alpha$-and $\beta$-dihydrodiphosphopyridine nucleotide. Curve $A, \alpha$ - and $\beta$-forms exeited at $340 \mathrm{~m} \mu$; curve $B, \beta$-form excited at $260 \mathrm{~m} \mu$, curve $C, \alpha$-form excited at $260 \mathrm{~m} \mu$ These values are relative since the intensities of the excitin light have not been determined intact $\beta$-isomer of dihydrophosphopyridine nucleo. tide, whereas this ratio becomes 3.4 in propylene glycol. A similar examination of the reduced $\alpha$-isomer reveals that the $E_{260} / E_{340}$ ratio is the same in water as in the propylene glycol.

Studies similar to those described for the $\alpha$-isomer of dihydrodiphosphopyridine nucleotide have been carried out with deamino-dihydrodiphosphopyridine nucleotide (the hypoxanthine analogue ${ }^{5}$ ) and with the 3-acetylpyridine analogue ${ }^{6}$. The latter analogue clearly showed the transfer of excitation energy since excitation at 260 mu gives a 478 -mu fluorescence ${ }^{-}$ which is also elicited by excitation at $365 \mathrm{~m} \mu$. The absorption spectrum of this analogue behaved much like $\beta$-dihydrodiphosphopyridine nucleotide in water and propylene glycol. The deamino-dihydrodiphosphopyridine nueleotide, however, did not show properties of energy transfer, since excitation of the purine ring did not result in fluorescence of the dihydropyridine nucleus.

In accordance with the postulate by Weber ${ }^{1}$ that the adenine and the dihydropyridine rings form a complex in $\beta$-dihydrodiphosphopyridine nucleotide, it appears that such complex formation cannot occur in the $\alpha$-isomer.

Further studies on the structural conformation of diphosphopyridine nucleotide and dihydrodiphosphopyridine nucleotide are being examined in this laboratory through the use of pyridine coenzyme analogues.

This work was supported by grants from the National Cancer Institute, National Institutes of Health (No. CY3611) and National Science Foundation Grants (No. G-4512 and No. G-6448).

\section{Sidney ShIFrin}

NathaN O. KaPlaX̃

Brandeis University, Waltham 54,

Mass.

March 10.

${ }^{1}$ Weber, G., Nature, 180, 1409 (1957).

${ }^{2}$ Kaplan, N. O., Ciotti, M. M., Stolzenbach, F. E., and Bachur, N. R., J. Amer. Chem. Soc., rig, 815 (1955).

${ }^{3}$ Haynes, L. J., Hughes, N. A., Kenner, G. W., and Todd, A., J. Chem. Soc., 3727 (1957).

4 Karrer, P. and Blumer, F., Helv, Chim, Acta, 30, 1157 (1947).

${ }^{5}$ Kaplan, N. O., Colowick, S. P., and Ciotti, M. M., J. Biol. Chem., 194, 579 (1952).

${ }^{6}$ Kaplan, N. O., and Ciotti, M. M., J. Biol. Chem., 221, 828 (1956)

'Shifrin, S., and Kaplan, N. 0., Proc. U.S. Nat. Acad. Sci., 44, 177 (1958).

\section{A Possible Negative Feedback Phenomenon controlling Formation of Alkaline Phosphomonoesterase in Escherichia coli}

WE have already reported ${ }^{1}$ that in a phosphatedeficient medium the amount of ribonucleic acid in $E$. coli decreased remarkably, while there was an appreciable increase of deoxyribonucleic acid, protein and viable cell numbers. At the same time we also studied activities of some enzymes presumed to be related to the degradation of ribonucleic acid, for example, ribonuclease phosphodiesterase and phosphomonoesterase, and found a marked increase in that of phosphomonoesterase. This communication is coneerned with this formation of phosphomonoesterase in $E$. coli.

The strain used was $E$. coli $B$ and the medium contained per litre: $3 \cdot 0 \mathrm{gm}$. $\mathrm{NaCl}, 0.25 \mathrm{gm}$. 